\title{
Cytogenetic analysis in infertile male patients with oligospermia and azoospermia in the southern region of Iran, Shiraz
}

\begin{abstract}
Background: Infertility was found to affect approximately $10 \%-15 \%$ of the couples, worldwide. Male factors are responsible for at least $50 \%$ of the infertility cases. The chromosomal abnormality is more common in infertile men than in fertile men. However, the aim of this study was to evaluate the frequency and type of major chromosomal abnormalities in the infertile men with problems in their sperm count, who had been referred to cytogenetic center in Shiraz, the main referral center in southern Iran.
\end{abstract}

Materials and Methods: A total of 433 infertile males, with azoospermia [169(31\%)] and oligospermia [264(69\%)], were included in this prospective observational study. Samples were retrospectively collected from the infertile males, and examined by karyotype analysis.

Results: The findings revealed that there are $17.3 \%$ chromosomal problems, in which $14.3 \%$ and $3 \%$ of all cases exhibited numerical and structural abnormalities, respectively. Among the 433 infertile patients, 57(33\%) exhibited a numerical sex chromosome abnormality, including 49 (11.3\%) subjects with typical Klinefelter syndrome, 4 (2.95\%) ones with structural sex chromosome and $11(4.4 \%)$ ones with the autosomal chromosome abnormality.

Conclusion: The results from this study demonstrated that chromosomal abnormalities are common in the infertile men with a higher frequency of sex chromosomal abnormality, especially those with the numerical type. This highlights the importance of karyotype findings to make appropriate decisions regarding the management of the patients in infertility clinics.

Keywords: infertility, cytogenetic analysis, azoospermia, oligospermia
Volume 6 Issue 3 - 2016

\author{
Vahab Rekabi,,'2 Ahmad Monabati,' Sadat \\ Noori, ${ }^{2}$ Akbar safaei ${ }^{2}$ \\ 'Department of pathology and hematology research center, \\ Shiraz University of Medical sciences, Iran \\ ${ }^{2}$ Department of pathology, molecular pathology and cytogenetic \\ ward, Shiraz University of Medical sciences, Iran
}

\begin{abstract}
Correspondence: Vahab Rekabi, Fellowship of molecular pathology and cytogenetic, Shiraz University of Medical sciences, Iran, Tel +989|23247864, Fax 7| 3230I784,
\end{abstract}

Email vahabrekaby@hotmail.com

Received: September 06, 2016 | Published: December 14, 2016

\section{Introduction}

Infertility, considered as one of the main public health issues, affects about $10 \%-15 \%$ of the couples in reproductive age. Male factors are known to be responsible for $50 \%$ of all infertilities. It is well-established that the chromosomal abnormality is more prevalent in infertile men than in fertile men. ${ }^{1}$ In developed countries, the incidence factor is considered to be less, when compared to underdeveloped countries. ${ }^{2}$ Male factor infertility includes chromosome and gene abnormalities, hormonal problems, genital infections, chemical and physical agents, varicose, genito-urinary obstruction, testicular dysfunction, etc. In addition, genetic abnormalities are suspected to result in male infertilities in about one-third of the cases, referred to as idiopathic.

There is a clear correlation between genetic abnormalities and infertility; some chromosomal aberrations are inherited, while others result from de novo mutations. This can lead to the reduction or even loss in sperm production. Importantly, the latter may result in a chromosomally-abnormal zygote, leading to either fetal death or the birth of an abnormal child. ${ }^{3}$ It should be noted that the primary mutational event in the stem cells could arise from structural abnormalities, including deletions, translocations or inversions, during mitotic and meiotic divisions. ${ }^{4}$ Male infertility work up is a complex process that should include a past medical history, physical examination, hormonal assay, semen count and analysis and karyotype..$^{5}$ It is rather established that there is an increase in the frequency of chromosomal abnormalities in infertile men, as compared to fertile men. Because there are various procedures for the cytogenetic analysis as well as clinically-heterogeneous patients, most studies have reported a wide range of chromosomal abnormal frequencies, ranging from 5.9 to $17.1 \%$, as shown in Table $2 .{ }^{6-12}$ In this light, the present study was mainly aimed to investigate the possible cytogenetic abnormalities that result in azoospermia and oligospermia in infertile men.

\section{Materials and methods}

A total of 433 patients with infertility problems were included in this cross-sectional study, and assessed retrospectively. All these cases, referred to Molecular Pathology and Cytogenetic ward of Department of Pathology, Shiraz University of Medical Science, Iran, during the 3-year period from June 2011 to August 2014, were included in the final analysis, except for patients without metaphase or karyotype information. The patients' age varied between 18 and 60 years old, with a mean age of 33.6 years old. Ethical approval was obtained from the Local Research Ethics Committee of Shiraz University of Medical Sciences; written informed consent was obtained from all study participants prior to enrolment into the study. The patients were divided into two groups, the Azoospermic group with the absence of sperm cells, and oligozoospermic group with sperm concentration of less than $20 \mathrm{million} / \mathrm{ml}$. The incidence of chromosomal abnormalities was investigated by the $G$ banding method in azoospermic and oligospermic infertile men. (Azoospermic group with 169 cases and oligospermic group with 264 cases). Although the chromosomal abnormalities exhibited various patterns in the oligozoospermia and azoospermia patients, the findings demonstrated that an increase in 
the frequency of chromosomal abnormalities was accompanied by a decrease in sperm count.

A failure to achieve pregnancy after 1 year of regular intercourse is considered as the criterion for infertility. A semen analysis according to the WHO guidelines (1999), Hormonal assay and sonography were carried out in all patients. The data from this study were analyzed by the SPSS 16 software. Some subjects with missing or incomplete information and an unavailable or no metaphase karyotype analysis were excluded from the study.

For cytogenetic analysis venous blood samples were collected into heparin syringes. The lymphocytes were cultured in RPMI 1640 basal media, supplemented with $10 \%$ FBS (Gibco, Invitrogen, USA), treated with $0.1 \mu \mathrm{g} / \mathrm{ml}$ colcemide (Gibco, Invitrogen, USA), and incubated under standard conditions in a humidified $5 \% \mathrm{CO} 2$ incubator at $37^{\circ} \mathrm{C}$ for 72 hours. Subsequently, the metaphase chromosomes were spread on marked glass slides, and stained by using the standard G-banding techniques. A minimum of about 15 cells with a normal metaphase spread of chromosomes were examined in each case. However, when mosaics were suspected, further metaphases were investigated. The karyotypes were interpreted according to the International System for Human Cytogenetic Nomenclature 2009, (ISCN) and analyzed CYTOVISION, a computer software to better diagnosis of chromosomal abnormality.

\section{Results}

As a whole, 169 and 264 patients were found to be Azoospermia and Oligospermia, respectively. Because this study was retrospective we haven't been normal control and all of cases referred for cytogenetics study. There is no clear association between sonography or hormonal results and chromosomal abnormality, suggesting that there were likely to be cases with incomplete information. It was found that there are 75 patients with the chromosome abnormality $(17.3 \%)$, from which $14.3 \%$ and $3 \%$ of all cases showed numerical and structural abnormalities, respectively. Out of 169 azoospermic males, 55 patients $(12.7 \%)$ showed the chromosomal abnormality. Interestingly, 54 cases were found to be the numerical abnormality, while $2(0.4 \%)$ cases exhibited the structural abnormality. However, there was an azoospermia case with the karyotype 47, XXY, inv(9), showing both structural and numerical types of chromosomal abnormality, as indicated in Table 1. Out of 264 oligospermic males, 15 patients showed karyotype abnormalities. Importantly, both kinds of chromosome abnormalities, structural and numerical, were detected among the cases, as shown in Table 1 .

Most of the numerically-abnormal patients had a Klinefelter karyotype (12.2\%) [49 patients (11.3\%) with a 47,XXY karyotype (Figure1), a $47, \mathrm{XXY}, \operatorname{inv}(9)$, and 3 patients $(0.7 \%)$ with mosaic consisting of a patient with $\operatorname{mos} 47, \mathrm{XXY} / 46, \mathrm{XY}(0.2 \%)$, one with $\operatorname{mos} 47, \mathrm{XXY} / 48, \mathrm{XXY} / 46, \mathrm{XY}(0.2 \%)$ and one with mos47, XXY/46, $\mathrm{XX} / 46 \mathrm{XY}(0.2 \%)]$. Other gonosomal-chromosome abnormalities were composed of 3 patients with the $47, \mathrm{XYY}$ syndrome $(0.7 \%)$, one with 48, XXYY $(0.2 \%), 2$ with 46, XY,inv (Y) $(0.5 \%)$ and one with $46, \mathrm{XY}, \mathrm{t}(\mathrm{Y} ; 19)(0.2 \%)$, as indicated in Figure 2.

The frequency of autosomal chromosome abnormalities detected in the present study was $2.5 \%$ (11/413 patients), consisting of a patient with 47,XXY,inv(9), 3 with 46,XY,inv(9), one with 45,XY,rob $(13 ; 15)$, one with $45, X Y, \operatorname{rob}(13 ; 21)$, one with $45, X Y, \operatorname{rob}(21 ; 22)$, one with $46, X Y, t(16 ; 20)$, one case with $46, X Y, t(2: 21)$, one case with mos $47, \mathrm{XY}+\operatorname{mar} / 46, \mathrm{XY}$, and a case with mos 46,XY,del(10)(q24)/46,XY, as illustrated in Table 1.

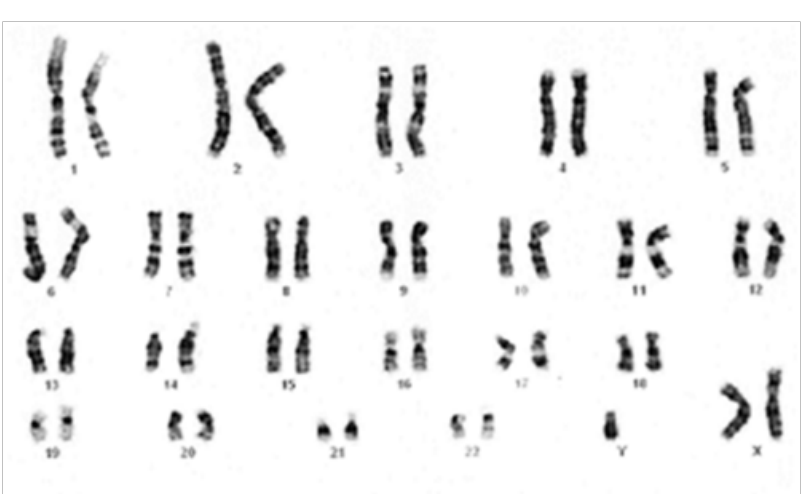

Figure I The Klinefelter karyotype features, $47, X X Y$ in a patient with azoospermia.

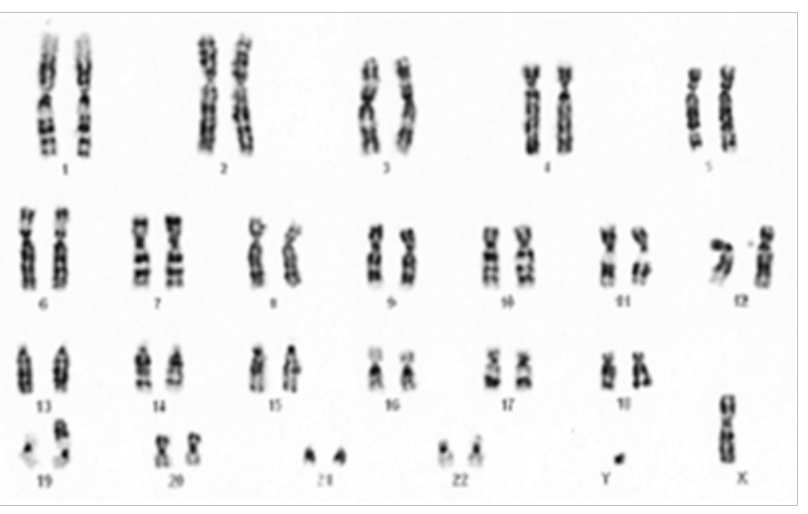

Figure $2 \mathrm{~A} 46, \mathrm{XY}, \mathrm{t}(\mathrm{Y} ; \mathrm{I9})(\mathrm{q} I \mathrm{I} ; \mathrm{p} \mid 3)$ karyotype in the patient with azospermia.

\section{Discussion}

In infertile males with severe spermatogenesis impairment, the chromosomal aneuploidy appears to be more common than other abnormalities with low sperm quality. ${ }^{6}$ There are numerous cytogenetic problems including numerical and structural abnormalities in both sex and autosomal chromosomes, composed of the gain or loss of a single chromosome, which in turn results in aneuploidy and structural abnormalities such as balanced or unbalanced translocations, inversion, etc. However, the above-mentioned abnormalities have been reported in several studies with different frequencies, ranging from 5.9 to $17.1 \%$ for infertile men (Table 2). ${ }^{6-12}$ The possible explanations for this various frequencies may be resulted from variation in the populations under study, geographical, environmental or genetic heterogeneities, or methodological detection problems for small statistical group. In the present study, $17.3 \%$ of the cases showed the chromosomal abnormality. Evaluation of 7 similar studies from the literature shows an average of $11.33 \%$ chromosomal abnormality rate. ${ }^{6-12}$ This difference may be due to a marked genetic variation in the different population, as illustrated in Table 3. Chromosomal abnormalities are more frequently found in the population of azoospermic and/or oligospermic males, when compared with general population. In our study, the most abnormal karyotype was observed in the patients with the azoospermia subgroup. In addition, chromosomal alterations were detected in 55 of 169 azoospermic cases and 15 of 264 oligozoospermic cases. Klinefelter's syndrome (KS) was the most type of the karyotype abnormality.

In the present study, multiple mosaic chromosomal abnormalities consisted of mos47, XXY/46, XY and mos47, XXY/46, XX/46, XY 
in the azoospermic subgroup and mos46, XY, $\operatorname{del}(10)(\mathrm{q} 24) / 46, \mathrm{XY}$, $\operatorname{mos} 47, \mathrm{XXY} / 48, \mathrm{XXYY} / 46, \mathrm{XY}, \operatorname{mos} 47, \mathrm{XY},+\operatorname{mar} / 46, \mathrm{XY}$ in the oligospermia cases have been seen.

Men with a 47, XXY karyotype are observed more frequently in infertile populations with azoospermia or severe oligozoospermia. In our study, the incidence of classic $\mathrm{KS}$ and its variants was $12.2 \%$, nearly similar to other previously studies. KS is the more common abnormality in sexual differentiation, observed in about 1 in 1000 live births. ${ }^{13}$ Interestingly, KS are recognized in tall young infertile men, testicular hypotrophy and elevated gonadotropin plasma levels, where gonadotropin is considered as one of the most important types of male hypogonadism in infertility clinics. ${ }^{14}$ In our study, it was found that all of the KS cases with a non-mosaic 47, XXY karyotype, as well as 2 of 3 cases with the mosaic form of KS belong to the azoospermic subgroup (Table 3).

Table I Chromosomal abnormalities among 433 infertile men studied

\begin{tabular}{|c|c|c|c|}
\hline Karyo type & Azoospermia (n/\%) & Oligospermia (n/\%) & Total (n/\%) \\
\hline $46, X Y$ & $114(26.3 \%)$ & $249(57.5 \%)$ & $363(83.82 \%)$ \\
\hline $47, X X Y$ & $49(11.3 \%)$ & 0 & $49(\mid 1.3 \%)$ \\
\hline 47,XXY,inv(9)(p||q|3) & I (0.2\%) & 0 & I $(0.2 \%)$ \\
\hline Mos $47, X X Y / 46, X Y$ & I (0.2\%) & 0 & I $(0.2 \%)$ \\
\hline Mos 47,XXY/48,XXYY/46,XY & 0 & $\mathrm{I}(0.2 \%)$ & I $(0.2 \%)$ \\
\hline Mos 47,XXY/46,XX/46,XY & I (0.2\%) & 0 & I $(0.2 \%)$ \\
\hline $47, X Y Y$ & I $(0.2 \%)$ & $2(0.5 \%)$ & $3(0.7 \%)$ \\
\hline $48, X X Y Y$ & I (0.2\%) & 0 & $\mathrm{I}(0.2 \%)$ \\
\hline $46, X Y$, inv $(Y)(p|| .2 q|| .2)$ & 0 & $2(0.5 \%)$ & $2(0.5 \%)$ \\
\hline $46, X Y, t(16 ; 20)(q 22 ; p \mid 3)$ & 0 & I $(0.2 \%)$ & I $(0.2 \%)$ \\
\hline $46, X Y, t(Y ; 19)(q|I ; p| 3)$ & I (0.2\%) & 0 & I $(0.2 \%)$ \\
\hline $46, X Y, t(2 ; 2 I)(p I I ; p I I)$ & 0 & $\mathrm{I}(0.2 \%)$ & I $(0.2 \%)$ \\
\hline $46, X Y, \operatorname{inv}(9)(p|2 q| 3)$ & 0 & $3(0.7 \%)$ & $3(0.7 \%)$ \\
\hline $45, X Y, \operatorname{rob}(21 ; 22)(q|0 ; q| 0)$ & 0 & $\mathrm{I}(0.2 \%)$ & I $(0.2 \%)$ \\
\hline $45, X Y, \operatorname{rob}(13 ; 15)(q|0 ; q| 0)$ & 0 & I $(0.2 \%)$ & I $(0.2 \%)$ \\
\hline $45, X Y, \operatorname{rob}(|3 ; 2|)(q|0 ; q| 0)$ & 0 & I $(0.2 \%)$ & I $(0.2 \%)$ \\
\hline Mos 46,XY, del(I0)(q24))/46,XY & 0 & $\mathrm{I}(0.2 \%)$ & I $(0.2 \%)$ \\
\hline Mos $47, \mathrm{XY},+\operatorname{mar} / 46, \mathrm{XY}$ & 0 & $\mathrm{I}(0.2 \%)$ & $\mathrm{I}(0.2 \%)$ \\
\hline Total & $169(39 \%)$ & $264(61 \%)$ & $433(100 \%)$ \\
\hline
\end{tabular}

Table 2 A comparison between pervious literature reviews of studies published during 2002 to 2009 with our present study

\begin{tabular}{lllllll}
\hline Authors & NO & Oligospermia & Azoospermia & Numerical & Structural & Frequency \\
\hline Vincent et al. $^{6}$ & $256 \mid$ & $93 / 1859(5 \%)$ & $1 \mathrm{II} / 792(14 \%)$ & $13 \mathrm{I}$ & 73 & $7.69 \%$ \\
Salahshourifar et al. $^{7}$ & 874 & $1 \mathrm{I} / 175(6.2 \%)$ & $106 / 444(23.8 \%)$ & 116 & 20 & $15.50 \%$ \\
samlet et al. $^{8}$ & 819 & $10 / 436(2.2 \%)$ & $42 / 383(10.9 \%)$ & 39 & 13 & $5.90 \%$ \\
Akgul et al. $^{9}$ & 179 & $5 / 73(17.4 \%)$ & $15 / 86(17.4 \%)$ & 16 & 2 & $11.74 \%$ \\
Nagvanker et al. $^{10}$ & 88 & $3 / 46(6.5 \%)$ & $6 / 42(14.3 \%)$ & 4 & 5 & $10.20 \%$ \\
Ebru Etem et al" & 214 & $5 / 76(6.5 \%)$ & $19 / 138(13.7 \%)$ & 18 & 6 & $11.20 \%$ \\
Larysa YP et al. ${ }^{12}$ & $29 \mid$ & $21 / 164(12.8 \%)$ & $14 / 40(35 \%)$ & 18 & 31 & $17.10 \%$ \\
our study & 433 & $15 / 264(5.6 \%)$ & $55 / 169(32.5 \%)$ & 62 & 13 & $17.30 \%$ \\
\hline
\end{tabular}

Table 3 Literature reviews, Azoo/oligospermia in the Klinefelter's syndrome, as compared with our study

\begin{tabular}{|c|c|c|c|}
\hline Study & Numbers of KS & Azoospermia & Oligozoospermia I 5 \\
\hline Ceylan et al..$^{15}$ & - & $90 \%$ & $10 \%$ \\
\hline Ferlin et al. ${ }^{16}$ & - & $90 \%$ & $10 \%$ \\
\hline P a Kosar et al. ${ }^{17}$ & 14 & $86 \%$ & $14 \%$ \\
\hline Larysa YP et al. ${ }^{12}$ & 10 & $90 \%$ & $10 \%$ \\
\hline Our study & 53 & $98 \%$ & $2 \%$ \\
\hline
\end{tabular}

Although structural autosomal abnormality that effaced on azoospermia/oligospermia patient not cleared on previous study we found numbers of this abnormality in cases with low sperm count. Pericentric inversion of the chromosome 9 usually considered as a normal variable feature of this chromosome in general population, but maybe appears to play some important roles in the infertility development. In the present study, 4 cases were found with pericentric $\operatorname{inv}(9)$, in which three patients with 46, XY, inv(9)(p12q13) and a $47, \mathrm{XXY}, \operatorname{inv}(9)(\mathrm{p} 11 \mathrm{q} 13)$ as a variant of KS were oligospermic and azoospermic, respectively.

A relationship between infertility and autosomal translocation has been observed among severely-oligo / azoospermic patients. In this 
study, reciprocal translocation $\mathrm{t}(16 ; 20), \operatorname{rob}(2 ; 21), \operatorname{rob}(13 ; 15)$ and $\operatorname{rob}(15 ; 21)$ were detected in oligospermic males, while $t(Y ; 19)$ did in one azoospermic male. The exact mechanism by which chromosomal abnormality induces infertility is not fully understood. While balanced reciprocal translocation governing two autosomal chromosomes is maybe associated with decreased sperm count, infertility and / or the increased risk of recurrent abortion, reciprocal translocation governing $\mathrm{Y}$ and an autosomal chromosome is usually associated with azoospermia. ${ }^{18}$ It is worthy to note that $\mathrm{Y} /$ autosome translocation may either affect heterochromatic or euchromatic parts of the $\mathrm{Y}$ chromosome. The latter case is usually resulted from a breakpoint in Yq11, and in most cases associated with infertility. ${ }^{18}$ Nevertheless, there is a reported case with $\mathrm{Y} /$ autosome translocation affecting the $\mathrm{Y}$ euchromatic part who has been found to be fertile. ${ }^{18}$

From the genetic basis of infertility point of view, it is estimated that most of all abnormalities take place in acrocentric chromosome, indicating their important role in male fertility. Guichaoua et al. ${ }^{19}$ explained the correlation between acrocentric chromosome involvement in infertile men and the severity of the spermatogenic defect. This is based on potential autosomal gene defects in these chromosomes affecting male gametogenesis. However, further investigation is recommended. Vincent et al. $^{6}$ showed that an autosomal chromosome with structural abnormalities was primarily observed in severe oligospermia, ${ }^{6}$ This study confirms this finding because of detection of five autosomal translocations in oligospermic males. Among numerous etiologic factors, genetic factors play an important role in male infertility. ${ }^{20}$ In our study, it is demonstrated that most of the patients were azoospermic, as well as $\mathrm{KS}$ was the most common type of chromosomal abnormalities (12.2\%), only found in this group of patients.

\section{Conclusion}

In conclusion, the data from this study reveal that chromosomal abnormalities are common in infertile men with higher frequency of sex chromosomal abnormality, especially those with the numerical type. This study emphasizes the importance of cytogenetic study in referral cytogenetics center to make appropriate decision for the management of the patients in infertility clinics. It is recommended to carry out more developed cytogenetic studies, such as FISH or PCR for AZFs, as well as SRY to better understand the cytogenetic abnormality. However, this was limitation of our study. It is also recommended to perform the perfect hormonal evaluation, testicular sonography as well as biopsy if necessary, because of their important role in the management of patients.

\section{Acknowledgments}

This study was financially supported by the Shiraz University of Medical Sciences, Shiraz, Iran. We appreciate from the staffs of the cytogenetic ward of the pathology department of Shiraz University of medical science also referral centers of Motahari Medical clinic. We also acknowledge Dr Azad, Mehdi Zamani, Nahleh Jamali, Marzieh Hoseini as well as the technician groups of cytogenetic ward.

\section{Conflicts of interest}

Authors declare there are no conflicts of interest.

\section{References}

1. Bhasin S, de Kretser DM, Baker HW. Clinical review 64: Pathophysiology and natural history of male infertility. $J$ Clin Endocrinol Metab. 1994;79(6):1525-1529.
2. Diemer T, Desjardins C. Developmental and genetic disorders in spermatogenesis. Hum Reprod Update. 1999;5(2):120-140.

3. Egozcue S, Blanco J, Vendrell JM, et al. Human male infertility: chromosome anomalies, meiotic disorders, abnormal spermatozoa and recurrent abortion. Hum Reprod Update. 2000;6(1):93-105.

4. Vutyavanich T, Piromlertamorn W, Sirirungsi W, et al. Frequency of Y chromosome microdeletions and chromosomal abnormalities in infertile Thai men with oligozoospermia and azoospermia. Asian J Androl. 2007;9(1):68-75.

5. Morel F, Douet-Guilbert N, Le Bris MJ, et al. Chromosomal abnormalities in couples undergoing intracytoplasmic sperm injection. A study of 370 couples and review of the literature. Int J Androl. 2004;27(3):178-182.

6. Vincent MC, Daudin M, De MP, et al. Cytogenetic investigations of infertile men with low sperm counts: a 25-year experience. J Androl. 2002;23(1):18-22.

7. Salahshourifar I, Gilani MAS, Masoudi NS, et al. Chromosomal abnormalities in Iranian infertile males who are candidates for assisted reproductive techniques. Iran J Fertil Steril. 2007;1(2):75-79.

8. Samli H, Samli MM, Solak M, et al. Genetic anomalies detected in patients with non-obstructive azoospermia and oligozoospermia. Arch Androl. 2006;52(4):263-267.

9. Akgul M, Ozkinay F, Ercal D, et al. Cytogenetic abnormalities in 179 cases with male infertility in Western Region of Turkey: report and review. J Assist Reprod Genet. 22009;6(2-3):119-122.

10. Nagvenkar P, Desai K, Hinduja I, et al. Chromosomal studies in infertile men with oligozoospermia \& non-obstructive azoospermia. Indian $J$ Med Res. 2005;122(1):34-42.

11. Etem EÖ, Yüce H, Erol D, et al. Cytogenetic analysis in infertile maleswith sperm anomalies. Marmara Medical Journal. 2009;22(3):217-224.

12. Pylyp LY, Spinenko LO, Verhoglyad NV, et al. Chromosomal abnormalities in patients with oligozoospermia and non-obstructive azoospermia. J Assist Reprod Genet. 200730(5):729-732.

13. Nussbaum RL, McInnes RR, Willard HF. Thompson \& Thompson genetics in medicine. $7^{\text {th }}$ ed. Philadelphia Saunders, Elsevier: USA; 2007. 585 p.

14. Lee YS, Cheng AW, Ahmed SF, et al. Genital anomalies in Klinefelter's syndrome. Horm Res. 2007;68(3):150-155.

15. Ceylan GG, Ceylan C, Elyas H. Genetic anomalies in patients with severe oligozoospermia and azoospermia in eastern Turkey: a prospective study. Genet Mol Res. 2009;8(3):915-922.

16. Ferlin A, Arredi B, Foresta C. Genetic causes of male infertility. Reprod Toxicol. 2006;22(2):133-141.

17. Kosar PA, Ozcelik N, Kosar A. Cytogenetic abnormalities detected in patients with non-obstructive azoospermia and severe oligozoospermia. J Assist Reprod Genet. 2010;27(1):17-21.

18. Pabst B, Glaubitz R, Schalk T, et al. Reciprocal translocation between Y chromosome long arm euchromatin and the short arm of chromosome 1. Ann Genet. 2002;45(1):5-8.

19. Guichaoua MR, Delafontaine D, Noel B, et al. Male infertility of chromosomal origin. Contracept Fertil Sex. 1993;21(2):113-121.

20. Akbari MT, Behjati F, Pourmand GR, et al. Cytogenetic abnormalities in 222 infertile men with azoospermia and oligospermia in Iran: Report and review. Indian J Hum Genet. 2012;18(2):198-203. 